\title{
Desarrollo de funciones ejecutivas, de la niñez a la juventud
}

\author{
Julio C. Flores-Lázaro ${ }^{1,2 *}$, Rosa E. Castillo-Preciado ${ }^{3}$ y Norma A. Jiménez-Miramonte ${ }^{3}$ \\ ${ }^{1}$ Hospital Psiquiátrico Infantil "Dr. Juan N. Navarro", SAP-INMEGEN, Institutos Nacionales de Salud-México \\ ${ }^{2}$ Posgrado-UNAM (México) \\ ${ }^{3}$ Facultad de Psicologia, Universidad de Guadalajara, Centro Universitario del Sur (México)
}

\begin{abstract}
Resumen: Las funciones ejecutivas se encuentran entre los procesos más complejos del humano; su desarrollo permite la conformación de diversas capacidades de control, y organización de la conducta y la cognición. En este artículo se presenta una extensa revisión de la literatura más relevante a nivel internacional sobre el tema. Se abarca el proceso de desarrollo desde la niñez hasta la juventud, analizando las características de desarrollo de las principales funciones ejecutivas. Se muestra que la hipótesis planteada por Victoria Anderson hace ya una década es correcta: las funciones ejecutivas se desarrollan de forma secuencial y curvilínea: un intenso progreso en la infancia, con una desaceleración a inicios de la adolescencia. También se analiza el efecto de otros factores adicionales a la edad como son el efecto de la escolaridad, los estilos parentales y el contexto cultural.
\end{abstract}

Palabras clave: Desarrollo funciones ejecutivas; infancia; adolescencia.

\begin{abstract}
Title: Executive functions development, from childhood to youthhood. Abstract: The executive functions are among the most complex cognitive processes in the human; during development they support the conformation of several capacities for organization and control of behavior and cognition. In this article an extensive review on the most relevant scientific literature is presented, with a developmental range from childhood to youthhood. An analysis on the developmental characteristics of the main executive functions is offered. The review indicates that the hypothesis stated by Victoria Anderson a decade ago, is accurate: the executive functions develops in a sequential form, with a curvilinear behavior: an intense progression during childhood, with slowness by early adolescence. Effects of additional factors like school level, parenting, and cultural context are also analyzed.

Key words: Executive functions development; childhood; adolescence.
\end{abstract}

\section{Introducción}

De forma progresiva diversos teóricos e investigadores han coincidido en que las funciones ejecutivas (FE) se encuentran entre los componentes más importantes para que el desarrollo infantil y adolescente sea exitoso (Diamond \& Lee, 2011); entre las FE más importantes se encuentran el autocontrol, la memoria de trabajo, la organización, la planeación, la solución de problemas, y la flexibilidad de pensamiento; capacidades primordiales para un adecuado aprendizaje académico desde la infancia temprana (Best, Miller \& Naglien, 2001). Las investigaciones sistematizadas sobre el desarrollo de las FE se han incrementado progresivamente en los últimos 15 años, lo que ha producido un número significativo de resultados; con esta información se puede construir un panorama inicial-básico sobre el estado del arte de este tema.

A pesar de que ya se cuenta en la literatura con decenas de artículos sobre esta temática, recientes trabajos de revisión y metanálisis advierten que aún no se ha conseguido construir un conocimiento básico sobre el desarrollo infancia-adolescencia, debido a que la mayoría de las investigaciones se han enfocado a momentos específicos del desarrollo, y no hacia una perspectiva amplia sobre el mismo (Best, Miller \& Jones, 2009; Romine \& Reynolds, 2005).

El planteamiento del desarrollo secuencial de las FE durante la infancia y la adolescencia propuesto por Victoria Anderson (2001) -algunas funciones ejecutivas se desarrollan más temprano y con mayor velocidad que otras- es ampliamente aceptado, ya que de forma progresiva se han encontrado evidencias de diverso tipo: neuropsicológicas-

* Dirección para correspondencia [Correspondence address]: Julio César Flores Lázaro, San Buenaventura No. 86 Col. Belisario Domínguez Deleg. Tlalpan, México, D.F. C.P. 14080 (México). E-mail: j_neuro@yahoo.com.mx cognitivas, y estadísticas- (Huizinga, Dolan, \& Van der Molen, 2006; Van Leijenhorst, Westenberg \& Crone, 2008). En general se ha encontrado que la mayoría de las FE presentan un desarrollo acelerado en la infancia, con una meseta que se consigue a principios-mediados de la adolescencia -con algunas pocas excepciones- (Anderson 2002; Best \& Miller, 2010; Diamond, 2002; Romine \& Reynolds, 2005).

Como característica general de la investigaciones en este campo destaca que se han abordado muy pocas FE, generalmente con muestras limitadas por rango de edad o por número de sujetos incluidos (por ejemplo: Armengol, 2002; Smidts, Jacobs \& Anderson, 2004; Matute et al., 2008), muy pocas de ellas han incluido muestras cercanas o mayores a 200 sujetos (por ejemplo: Anderson et al., 2001). Un extenso metanálisis realizado a 20 años (Romine \& Reynolds, 2005) encontró que solo se pueden comparar estadísticamente cinco FE: flexibilidad, fluidez verbal, mantenimiento de set, fluidez de diseño y planeación secuencial. Sin embargo se ha señalado que ninguna de estas medidas son suficientemente representativas de la amplia variedad de FE: este metanálisis no pudo incluir capacidades como detección de selecciones de riesgo, procesamiento riesgo-beneficio, entre otras. Lo que de acuerdo a especialistas del campo, aún representa un panorama muy parcial del desarrollo (Zelazo \& Cunningham, 2007). En una de la revisiones más extensas y recientes (Best \& Miller, 2010) se establece que la mayoría de los artículos se han enfocado en momentos específicos de desarrollo (por ejemplo la edad preescolar, o la niñez), siendo escasos los artículos que han estudiado el desarrollo a lo largo de la dimensión infancia-adolescencia. Estos mismos autores señalan que el principal obstáculo para estudiar esta dimensión es la falta de instrumentos de evaluación específicamente diseñados para que sus pruebas-reactivos permitan realizar análisis comparativos a través de las distintas edades.

Por la imposibilidad de comparar el desempeño de niños preescolares con infantes y adolescentes (debido principal- 
mente al uso de pruebas distintas), en las principales revisiones se ha determinado no incluir el análisis de los resultados de preescolares (para una revisión extensa de esta etapa ver: Garon, Bryson \& Smith 2008).

Enfoque conceptual: se ha propuesto que las FE conforman un sistema, el cual se desarrolla desde funciones aisladas hasta la integración compleja entre ellas (Zelazo, Mulller, Frye \& Marcovitch 2003). A su vez diversos investigadores han encontrado un desarrollo "piramidal", en el que FE más básicas -por ejemplo control inhibitorio- preceden y soportan el desarrollo de FE más complejas -por ejemplo memoria de trabajo y flexibilidad mental- (Best et al., 2009; Huizinga et al., 2006; Romine \& Reynolds, 2005). Así a lo largo del desarrollo, la integración entre diversas FE es lo que permite el desarrollo complejo de la competencia ejecutiva (Zelazo \& Frye, 1997). En este artículo se proporcionará un panorama individual de las FE, intentando aportar un marco conceptual para su integración.

Procedimiento para la revisión: en el marco del metanálisis de Romine y Reynolds (2005), de la revisión de Anderson (2002) y la extensa revisión de Best y Miller (2010), se seleccionaron (del libro Desarrollo neuropsicológico de lóbulos frontales y funciones ejecutivas, Flores Lázaro y Ostrosky Shejet, 2012) de forma cualitativa, los artículos más representativos de los principales grupos de investigación -en el contexto internacional- en este campo en los últimos 15 años. Originalmente se identificaron 153 artículos, de los cuales se seleccionaron 74 por su representatividad conceptual, para lograr una perspectiva general del desarrollo infancia-adolescencia.

De acuerdo a la propuesta de Best \& Miller (2010) la información obtenida se dividió para su presentación en etapas: desarrollo muy temprano (niñez temprana), desarrollo temprano (niñez tardía), desarrollo intermedio (adolescencia inicial-media) y desarrollo tardío (adolescencia tardíajuventud).

\section{FE de desarrollo muy temprano}

\section{Detección de selecciones de riesgo}

La prueba de cartas Iowa desarrollada por Bechara Tranel, Damasio \& Damasio (1996), permite evaluar el tipo de selecciones que un sujeto hace en base a la determinación implícita de una probable recompensa o de un probable castigo (Elliot et al., 2000). Para investigar sobre el desarrollo del procesamiento riesgo-beneficio se ha tenido que adaptar la prueba original a las características cognitivas y psicológicas de los niños. Los principales grupos que han investigado sobre este proceso en preescolares han encontrado que la capacidad de detección de selecciones de riesgo (selecciones que representan pérdidas o castigos) presenta desempeños competentes desde edades tan tempranas como los 4-5 años (Crone \& Van der Molen, 2004; Crone, Bunge, Latenstein \& van der Molen, 2005; Kerr \& Zelazo, 2003; Zelazo \& Muller, 2002). Al comparar grupos de sujetos de distintas edades, se ha encontrado que desde los ochos años, lo niños son tan competentes como los adolescentes y los adultos para detectar selecciones de riesgo -en este tipo de paradigmas(Hooper et al., 2004; Overman et al., 2004; van Leijenhorst et al., 2008).

Estos hallazgos presentados en la literatura científica pueden parecer contra-intuitivos con el cliché generalizado de que los adolescentes "no miden el riesgo porque su corteza frontal es inmadura". Los hallazgos concretos sobre la capacidad de detección del riesgo indican que esta competencia se encuentra de forma muy temprana, pero que en la adolescencia la valencia motivacional de la recompensa es muy alta (por una mayor actividad del cuerpo estriado y del sistema dopaminérgico de recompensa), lo que hace que los adolescentes presenten conductas de riesgo aún cuando son suficientemente sensibles al mismo (Romer et al., 2011). Diversos investigadores han destacado que la conducta de riesgo en la adolescencia es un fenómeno complejo que no puede reducirse solamente a la capacidad de detecciones de selecciones de riesgo y menos al neurodesarrollo de una región específica del cerebro. Se ha mostrado ampliamente que el proceso de toma de decisiones en contextos de riesgo involucra diversas regiones cerebrales y diversos procesos cognitivos, psicológicos y psico-sociales (Crone, 2009).

\section{FE de desarrollo temprano}

\section{Control inhibitorio}

La capacidad de control inhibitorio refleja el dominio sobre las activaciones automáticas como una forma preferencial de procesamiento. Por medio de pruebas de efecto Stroop adaptadas, se ha encontrado que las activaciones automáticas de procesamiento para objetos con colores prototípicos se presentan desde la edad de 4 años; cuando los niños tienen que denominar objetos con colores distintos a su prototipo (por ejemplo un oso color verde, en lugar de color marrón) (Prevor \& Diamond, 2005). La automatización de la lectura de palabras se alcanza alrededor de los 7 años (Wright \& Wanley, 2003), los mecanismos de control inhibitorio sobre las respuestas de procesamiento automatizadas (denominar un color en lugar de leer una palabra) alcanzan su máximo desempeño entre los 9-10 años de edad (Best \& Miller, 2010), a partir de estas edades no se han encontrado diferencias significativas con el desempeño de adolescentes ni adultos jóvenes (León-Carrión, García-Orza \& PérezSantamaría, 2004; Wright \& Wanley, 2003). El desarrollo del control inhibitorio permite a los niños escolares realizar tareas mentales que requieren procesar información que compite entre sí (por ejemplo: seleccionar entre varias alternativas la mejor respuesta), permitiendo inhibir respuestas impulsivas que no son optimas, de esta forma los niños pueden darse tiempo para analizar y seleccionar la mejor respuesta posible, o el procedimiento más optimo para resolver una tarea. En el campo del desarrollo semántico, se ha encontrado que la construcción inicial de la capacidad de clasificación 
por analogías (semejanzas) presenta un desarrollo muy importante entre los 8 y 14 años, debido al progresivo desarrollo de una mayor capacidad (incluido el control atencional) para discriminar-analizar perceptualmente rasgoscaracterísticas de los objetos que se comparan (Thibaut, French \& Vezneva, 2010). Diversas evidencias similares han provocado que en años recientes se haya rescatado el planteamiento de Vigotsky sobre la importancia de la interdependencia-integración vs el desarrollo individual de cada proceso, como uno de los principales mecanismos del desarrollo psicológico (Scerif, 2010).

\section{FE de desarrollo intermedio}

La mayoría del conocimiento que se tiene sobre el desarrollo de FE proviene de la conducta curvilínea que presentan las FE de desarrollo intermedio: un incremento lineal en la infancia, con una transición curvilínea que termina en una meseta en la adolescencia (Anderson, 2002; Romine \& Reynolds, 2005).

\section{Memoria de trabajo}

Algunos autores han encontrado que el incremento en la capacidad de retención de dígitos en orden progresivo entre los 7 y 13 años no es significativo, en tanto que el incremento en la retención de dígitos en orden inverso se incrementa significativamente al doble en este mismo rango de edad; señalándose que este desempeño puede reflejar el desarrollo diferenciado entre mecanismos de secuenciaciónordenamiento y mecanismos de mantenimiento de la información contenida en la memoria de trabajo (Diamond, 2002). También se ha encontrado que la capacidad de memoria de trabajo viso-espacial secuencial (reproducir en un orden específico elementos visuales contenidos en la memoria de trabajo), alcanza su máximo desempeño alrededor de los 12 años de edad (Luciana \& Nelson, 2002). Sin embargo si la información sólo tiene que ser sólo mantenida (no manipulada en un orden secuencial) se alcanza desempeño adulto desde los 9 años, sin diferencias significativas hasta los 20 años (Luciana, Conklin, Hooper \& Yarger, 2005).

\section{Flexibilidad mental}

La capacidad para inhibir una estrategia cognitiva o secuencia de acción (evitar la perseveración), que permita generar una respuesta alternativa (flexibilidad mental), posibilita enfrentarse de forma eficiente y flexible (desde diversos ángulos) a la solución de problemas o a la presentación de contingencias, sobre todo a aquellas que cambian de forma constante o se presentan de forma imprevista. Esta capacidad se desarrolla de forma gradual durante la infancia y alcanza su máximo desempeño alrededor de los 12 años (Anderson, 2001; Cinan, 2006). El control de esquemas de acción y/o pensamiento, posibilita la generación de alternativas nuevas para solucionar problemas o mejorar el desem- peño cognitivo; capacidad esencial en el desarrollo cognitivo y en el desempeño académico.

\section{Planeación visuoespacial}

La capacidad de planeación viso-espacial (desempeño en laberintos) se ha relacionado con un óptimo desempeño ambiental. El desarrollo de esta capacidad es muy marcado y acelerado en la infancia y su desempeño máximo se alcanza a partir de los 12 años (Levin, Song, Swing-Cobbs \& Roberson, 2001).

\section{Memoria estratégica}

La capacidad para controlar la memoria de forma estratégica depende principalmente del control ejecutivo (Fernandez-Duque, Baird \& Posner, 2000). La memorización estratégica se encuentra entre las capacidades de control cognitivo más importantes (De Marie \& Ferron, 2003; Siegler, 2004), esta capacidad requiere la implementación de esfuerzo mental y de recursos cognitivos dirigidos a mejorar el desempeño de la capacidad de memorización-aprendizaje (Bjorklund, Dukes \& Douglas, 2009). Además del control ejecutivo se requiere de la capacidad de metacognición (Roebers, von der Linden, \& Howie, 2007). El control metacognitivo se ejerce a partir del conocimiento de las capacidades propias -monitoreo- (Nelson \& Narens, 1994); de esta forma los procesos cognitivos (lenguaje, memoria, pensamiento, etc.) pueden ser óptimamente controlados y mejorados (Shimamura, 2000).

Se considera que la aparición y desarrollo en la implementación de estrategias de memoria (memoria estratégica) es uno de los componentes más importantes de la memoria. Se ha descrito en la literatura que la niñez es el periodo de mayor intensidad de desarrollo para la memoria declarativa (Schneider, 2002); siendo los principales factores de este progreso el monitoreo y el control (Ghetti, Lyons, Lazzarin \& Cornoldi, 2008). A partir de los 7 años de edad los niños pueden implementar estrategias de memorización de una forma eficiente (Roebers, 2006), sin embargo la eficiencia en la implementación es más claramente observada hasta los 8 años de edad (Lange, Guttentag \& Nida, 1990; Schneider, Kron, Hünnerkopf \& Krajewski, 2004). Durante el desarrolo los niños aprenden de forma consciente a implementar estrategias para mejorar los procesos de memorización y aprendizaje, lo que incrementa de forma considerable su aprovechamiento escolar (Koriat, Goldsmith, Schneider \& Nakash-Dura, 2001; Schlagmuller \& Schneider, 2002). Por ejemplo alrededor de los 10-11 años de edad la agrupación semántica ya es evidente en las tareas de codificación de palabras (Bjorklund, Dukes \& Brown, 2009). El proceso general de desarrollo es lento pero constante a lo largo de la infancia, alcanzando su máximo desempeño alrededor de los 12 años de edad (Roebers, 2006).

El control estratégico de los procesos cognitivos es un proceso multifacético que incluye en un continuo: construir 
una estrategia, producirla de forma optima, y beneficiarse de ella (Miller, 2000). Aunque son interdependientes, estos tres componentes varían durante el desarrollo, la implementación estratégica no siempre es estable o productiva, depende del tipo de tarea-proceso cognitivo, no puede ser definida como una capacidad general, ya que los niños pueden implementar una estrategia de forma eficiente en una tarea y no hacerlo en otra (Waters, 2000). La variabilidad en la implementación estratégica es la regla en lugar de la excepción en los niños (De Marie \& Ferron, 2003). La capacidad para desarrollar estrategias efectivas de memoria no sólo impacta en un mejor desempeño durante la evocación (por ejemplo para contestar un examen), sino también permite discriminar entre la información que es relevante para memorizar, lo que potencia el aprendizaje selectivo del material que es semánticamente significativo. Todavía a los 8 años de edad, el agrupamiento semántico (semantic-clustering) no se observa de forma clara, es hasta los 10-11 años que se observa de forma estable (Bjorklund et al., 2009). El agrupamiento semántico se convierte en una de las estrategias más efectivas y utilizadas en adolescentes y adultos.

\section{Planeación secuencial}

La selección y secuenciación de esquemas de acción para resolver un problema que requiere de realizar movimientos contra-intuitivos (que aparentemente van en sentido "contrario" a la solución) y ordenados de forma secuencial, conforman la capacidad de planeación secuencial (Goldberg, 2001; Luria, 1986). Los "retrocesos" representan pasos de preparación que solo se pueden entender dentro de una secuencia de movimientos (submetas) enfocadas en el logro de un objetivo a mayor plazo (van de Heuvel et al., 2003). Los resultados obtenidos con el problema de tres discos de la prueba Torre de Hanoi indican que el desempeño máximo se alcanza hacia los 15 años de edad, indicando que de las FE intermedias es la que más tarde alcanza su máximo desarrollo. Estos resultados se han encontrado con los problemas de tres discos, ya que con los problemas de cuatro discos no se han encontrado diferencias significativas en la infancia (Welsh, 1991); esto se debe al alto número de movimientos necesarios para realizar la tarea, ya que se han identificado hasta 54 "rutas estratégicas" para resolver el problema de 4 discos, de las cuales 33 son indirectas y solo 11 son directas (Garber \& Golden-Meadow, 2002).

En conjunto, el desarrollo de estas FE de desarrollo intermedio permiten a los niños escolares procesar y manipular de forma mental una mayor cantidad de información, lo que posibilita la construcción temporal de esquemas mentales, permitiendo una adecuada comprensión e identificación de las condiciones más relevantes de las tareas/problemas dados (Richland, Morrison \& Holyoak, 2005). Posibilita el uso eficiente de estrategias de memoria-aprendizaje, entre ellas el agrupamiento; permite el desarrollo de diversas hipótesis de trabajo-alternativas de solución a problemas, y posibilita un importante avance en la organización y planeación de sus actividades escolares y personales (por ejemplo: planear lo que se hará y cómo se hará en el transcurso de una semana; qué materiales se requieren, cuantas personas, y que tiempo (horas-días) conlleva realizar una tarea escolar; qué actividades realizar antes de otras, para optimizar recursos/esfuerzos).

\section{FE de desarrollo tardío}

\section{Fluidez verbal}

Existen diversos estudios sobre el desarrollo de la fluidez verbal, por ejemplo Matute et al. (2004) encontraron que la fluidez verbal-semántica presenta incrementos continuos al menos hasta los 14-15 años de edad. Sin embargo los estudios sobre la fluidez de verbos son muy escasos; Flores Lázaro, Tinajero Carrasco \& Castro Ruiz (2011) encontraron que la fluidez de verbos presenta incrementos en su desempeño posterior a la adolescencia sólo si los sujetos continúan su preparación educativa en el nivel universitario.

En los niños de 5 a 7 años predominan los verbos de alta frecuencia como correr, saltar, caminar los cuales denominan acciones corporales; a partir de estas evidencias se ha planteado que la representación mental de los verbos se desarrolla en base al modelo de la teoría de la cognición "encarnada" (embodied cognition), la cual plantea la importancia de las experiencias sensorio-motrices en el desarrollo, representación y uso de los verbos (Kemmerer \& González Castillo, 2010). Cuando los niños se acercan a los 12 años de edad, los verbos abstractos empiezan a dominar en su repertorio expresivo (Ávila, 2004); situación que coincide con el desarrollo de la actitud abstracta para la generación de categorías semánticas (Flores Lázaro \& Ostrosky-Solís, 2008), la cual se explica en el siguiente apartado.

La adquisición de verbos es un proceso más complejo y prolongado que para los sustantivos (Jia, Kohnert, Collado \& Aquino García, 2006), a los 5 años de edad la expresión de verbos en infinitivo no se encuentra presente, utilizando con más frecuencia la forma en tercera persona ( $\mathrm{D}^{\prime}$ amico, Devescovi \& Bates, 2001). Se piensa que los verbos conforman una red semántica menos diferenciada que los sustantivos (en forma de matriz difusa), en donde el fenómeno de "intencionalidad" sería el nodo aglutinador principal (Viglioco Vinson, Damian \& Levelt, 2002). Por lo que las categorías de verbos que pertenecen a diferentes campos semánticos serían más difusas que para el caso de los sustantivos (Masterson et al., 2008), por lo que la generalización lexical y morfológica, así como el proceso de conformación de las redes semánticas para los verbos es más difícil de establecer. Debido a que los verbos difieren en el número y tipo de argumentos (roles de participación) que requieren: los intransitivos requieren sólo un argumento (el niño duerme), los transitivos requieren un sujeto y un objeto (Pedro pateó la pelota), etc.; cuando un verbo es procesado, se co-activan todos los argumentos que este posee, por lo que un verbo con múltiples argumentos tomará más tiempo en ser proce- 
sado que un verbo con un sólo argumento (Thordadottir \& Weismer; 2002); a mayor complejidad argumentativa del verbo, mayores opciones (co-activaciones) para analizar y seleccionar (selectividad en la recuperación); lo que conlleva a mayor costo de procesamiento (mayor uso de recursos cognitivos). Se requiere de una importante capacidad de análisis comparativo, de decisión y de selección cognitiva (denominada: mapeo), por lo que se ha planteado una muy importante dependencia del sistema de funciones ejecutivas en el uso activo de los verbos (Spalek \& Thompson-Schill, 2008).

Desde el enfoque psicolingüístico se plantea que los verbos codifican estructuras de argumento que activan marcos de procesamiento sintácticos y semánticos, que van más allá de la denominación concreta de una acción (Kremmer \& González Castillo, 2010). El hecho de que la fluidez verbal sea una tarea de producción de lenguaje y que la expresión y redacción verbal sean habilidades que se demandan de forma progresivamente compleja a medida que los niveles escolares aumentan y sobre todo a nivel universitario, puede reflejar una influencia directa del desarrollo de capacidades expresivas de lenguaje oral sobre la fluidez verbal, tal como se ha encontrado en estudios comparativos de fluidez verbal en sujetos con distintos grados de escolaridad (Ramírez, Ostrosky-Solís, Fernández \& Ardila, 2005). También se ha encontrado que los verbos actúan como unidades prototípicas para sintetizar categorías con mayor significado, permitiendo una simplificación sintáctica y semántica con un mínimo de pérdida de información (Thordardottir \& Weismer, 2001), de esta forma la fluidez verbal puede estar mucho más influenciada que otras FE por influencias lingüísticas, psicolingüísticas y cognitivas, pudiendo ser más sensible a las condiciones de escolaridad y a las demandas de procesamiento cognitivo de este tipo de ambientes.

\section{Abstracción y actitud abstracta}

La capacidad para categorizar es un factor fundamental para el desarrollo, particularmente la evolución desde el pensamiento y procesamiento concreto hacia el procesamiento abstracto presenta diversas fases de desarrollo en los niños, las cuales influencian y estructuran su procesamiento cognitivo (Gelaes \& Thibaut, 2006). Los niños preescolares frecuentemente comparan y categorizan objetos en base a rasgos perceptuales tales como su forma. Cuando de forma progresiva van aprendiendo nuevos conceptos y palabras que representan atributos semánticos-abstractos, prefieren utilizar estos conceptos en lugar de los criterios perceptuales (Gentner \& Namy, 2000). Ya durante el proceso de comparación entre objetos y eventos los niños van introduciendo de forma sistemática cambios y variantes en sus representaciones semánticas; estos cambios permiten transformar y construir experiencias en categorías semánticas definidas; lo anterior se consigue por medio de un análisis continuo, que permite la definición y redefinición de nuevas características semánticas y sobre todo los límites clasificatorios de estas categorías; sintetizándolas en estructuras conceptuales cada vez más precisas (Borodistky, 2007).

Se ha establecido una división conceptual entre el sistema de representación de conocimiento, y el uso activo y eficiente de esta información (Delis, Squire, Bihrle \& Massman, 1992; Miller, 2000). Esta división representa dos sistemas cognitivos distintos: el saber y el saber-hacer (Bright, Moss \& Tyler, 2004; Noppeney \& Price, 2002). La experiencia tiene un importante efecto en el desarrollo de la categorización, llegando en los niños de mayor edad a influenciar de forma principal la percepción y representación, por lo que las propiedades abstractas-categoriales de un objeto dominan sobre sus propiedades preceptúales, y con frecuencia estas representaciones son reubicadas en base a la adquisición de nuevas categorías (Boroditsky, 2007). Debido a esto durante la infancia los niños constantemente comparan objetos y los integran en categorías que transitan desde los rasgos preceptúales hasta rasgos abstractos hacia el final de la niñez (Gelaes \& Thibaut, 2006).

En diversas investigaciones se ha encontrado que en los adultos normales (escolarizados) el proceso se ha automatizado y es característico que se analice la información de manera abstracta, a pesar de haberse instruido a los sujetos para que "conscientemente" se fijen en rasgos concretos o perceptuales (Noppeney \& Price, 2002), a este fenómeno se le ha denominado actitud abstracta (Lezak, 2004). La predisposición de procesar información de manera abstracta se evalúa cuando se le pide a los niños-sujetos generar categorías (sin especificar el tipo de categoría que se espera), de esta forma se evalúa la tendencia del sujeto a utilizar la información abstracta (saber) para construir categorías (saber/hacer), el hallazgo más interesante es que los niños poseen ("saben") mucha más información abstracta que la que utilizan (saber/hacer) al resolver tareas-problemas. La generación de categorías semánticas abstractas presenta incrementos lineales constantes desde los 6 años de edad hasta la juventud; es ya en la adolescencia que se observa una dominancia de la actitud abstracta como la forma principal de categorización (Flores Lázaro \& Ostrosky-Solís, 2008), pero sólo cuando los sujetos se encuentran en contextos escolarizados (Flores Lázaro et al., 2011). Lo que indica que la actitud abstracta es una de las FE con mayor longitud de desarrollo y acompaña el desarrollo cognitivo de los sujetos desde la enseñanza primaria hasta la universitaria. En un apartado siguiente se tratará el efecto de la actividad y el nivel escolar sobre las FE.

La capacidad de abstracción es una de las capacidades más importantes en el desarrollo cognitivo, ya que permite a los niños transitar desde el procesamiento sensorialperceptual hacia el abstracto. La mayoría de los contenidos escolares representan esquemas semánticos abstractos que se van complejizando de forma progresiva, por lo que el prolongado desarrollo de la actitud-predisposición para analizar información de forma abstracta, representa uno de los principales precursores del desarrollo cognitivo complejo (Gentner \& Namy, 1999). 


\section{Resumen de las características de desarrollo}

Hasta el momento la literatura analizada indica que las FE que presentan un desarrollo más consolidado en la infancia temprana son la detección de selecciones de riesgo y el control inhibitorio; en la infancia tardía la memoria de trabajo, la flexibilidad mental, memorización estratégica, y la planeación visoespacial, presentan los desarrollos más significativos; en la adolescencia tardía se termina de desarrollar la capacidad de planeación secuencial; la fluidez verbal y la abstracción continúan su desarrollo en la juventud, pero sólo en contextos escolarizados.

La literatura revisada sugiere que el desarrollo cognitivo y psicológico del niño transita desde el control cognitivo de las respuestas impulsivas-emocionales, hacia un procesamiento cognitivo más selectivo; posteriormente hacia una mayor capacidad para mantener y manipular (procesar) información de forma mental, y hacia la generación de mayores y mejores hipótesis/alternativas/estrategias de solución de problemas/procedimientos, junto con un importante desarrollo en el aprendizaje y uso activo de estrategias de memoria (por ejemplo: establecer enlaces semánticos para el aprendizaje); en un fondo de un constante desarrollo de la capacidad de abstracción, y de una mayor competencia psicolingüística, lo que genera no solo un pensamiento eficiente sino más abstracto y psicolingüísticamente más complejo.

En la Figura 1 se presentan las secuencias de desarrollo para las distintas FE analizadas en el texto.

En la Figura 2 se presenta los tipos curvilineales de desarrollo que sugiere la literatura para las distintas FE analizadas. En esta figura se observa la condición universal sobre el desarrollo no-lineal de los procesos cognitivos (TuckerDrob, 2009), la visión simplista de que un proceso cognitivo simplemente "se hace mejor", debe ser superada por el conocimiento específico de las particularidades (enfoque curvilineal) de desarrollo de cada proceso, ante los distintos momentos-edades de desarrollo.

Por medio de diversos estudios cognitivos con modelos estadísticos (análisis de ruta, ecuaciones estructurales, etc.) se ha encontrado que el desarrollo de las diversas FE presenta un desarrollo piramidal, particularmente en la infancia (Overman et al., 2004; Brocki \& Bohlin, 2004), con relaciones que cambian a través de las edades. Se propone que de forma progresiva durante el desarrollo, FE más básicas soportan FE más complejas, manteniendo una relación piramidal durante la niñez (Best et al., 2009; Huizinga et al., 2006; Romine \& Reynolds, 2005).

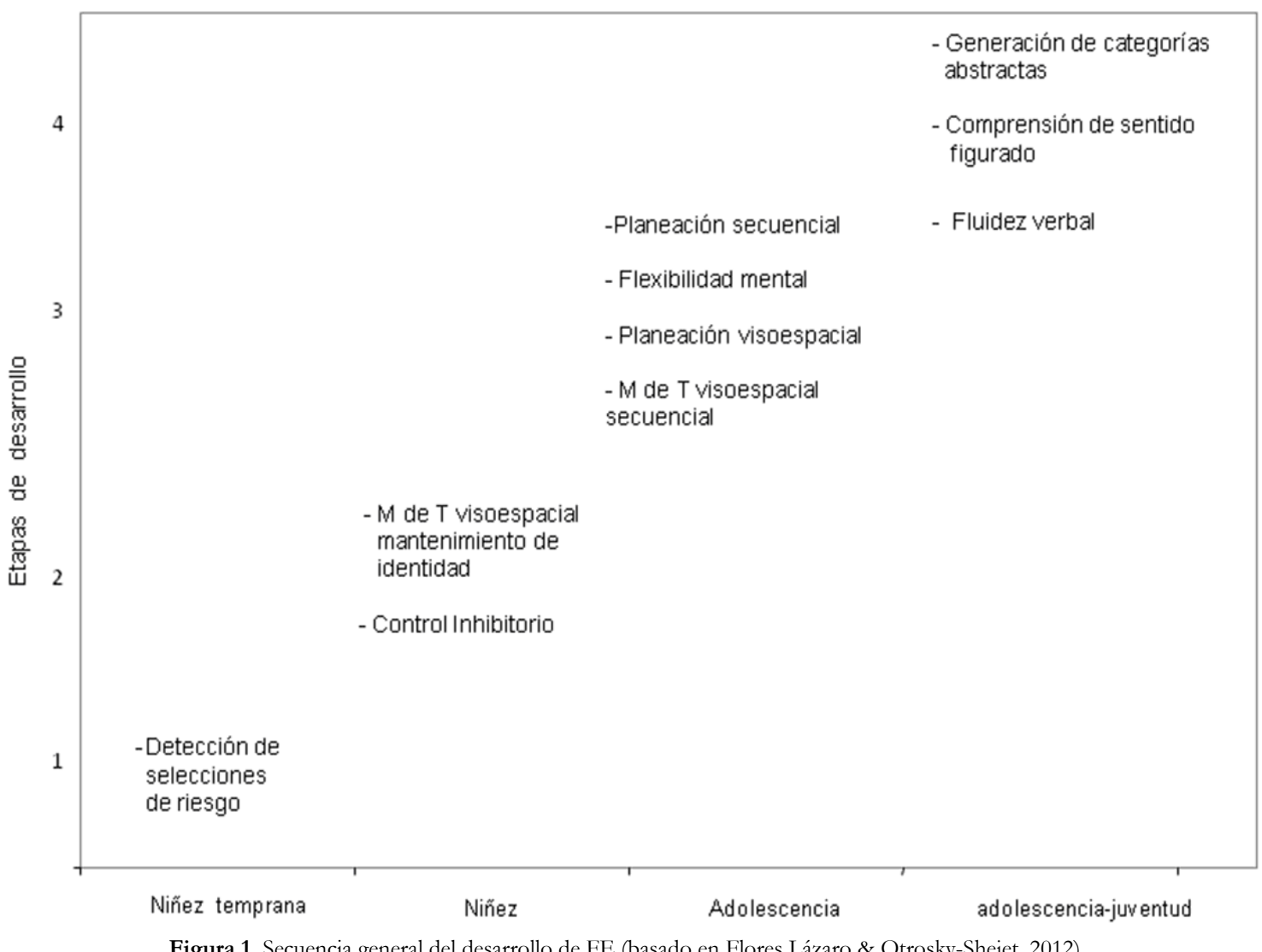




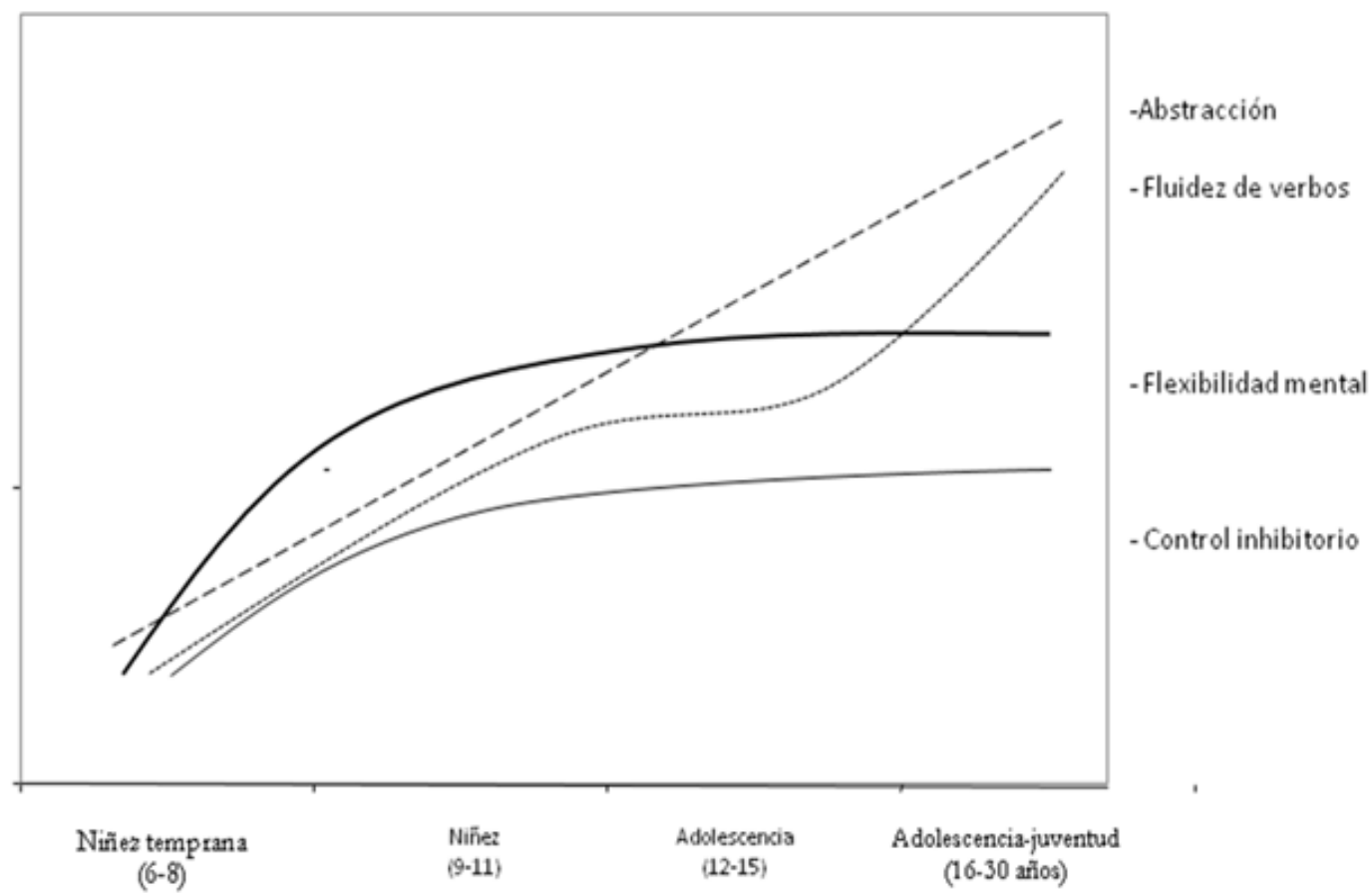

Figura 2. Tipos de conductas de las FE durante el desarrollo (basado en Flores Lázaro \& Otrosky-Shejet, 2012).

Durante la adolescencia ocurre una importante reorganización de las FE (Anderson, 2001; Rosso, Young, Femia \& Yurgelun-Todd, 2004), hacia la juventud se ha encontrado que se mantiene cierta independencia entre ellas: varios factores relacionados con distintas FE, en lugar de pocos factores que expliquen diversas FE (Pineda, Merchan, Roselli \& Ardila; 2000).

Los modelos integrativos imponen la necesidad de ir más allá de la división por procesos, implicando que la relación entre los diversos procesos cognitivos es por sí misma objeto de estudio, y más importante aún: presentan propiedades y características de desarrollo que pueden ser estudiadas en sí mismas (Best \& Miller, 2010). Sin embargo el análisis sobre la determinación de las FE de dominio-específico vs las FE de dominio-general aún se encuentra en desarrollo (Best et al., 2009). En uno de los estudios más importantes sobre desarrollo cognitivo (no específico de FE), se encontró que los procesos cognitivos tienden a integrarse-relacionarse más durante la niñez, sin embargo durante la juventud-adultez algunos de ellos tienden a diferenciarse; se piensa que esta diferenciación permite además de un control jerárquico más claro, una mayor flexibilidad-repertorio de opciones de respuestas (Tucker-Drob, 2009).

\section{Desarrollo cognitivo y FE}

Para contextualizar el desarrollo cognitivo desde una perspectiva integrativa se ha propuesto el modelo de complejidad creciente (Zelazo \& Frye, 1997). Se basa en la aplicación del modelo de sistema funcional (Luria, 1986), a la comprensión y estudio del desarrollo (Zelazo \& Muller, Frye \& Marcovitch 2003). Propone abordar las FE como un sistema funcional con múltiples sub-componentes, él cual está basado en resultados. Durante el desarrollo cada uno de los subcomponentes se desarrolla a su propio ritmo, pero finalmente la integración funcional basada en resultados da cuenta de un sistema que se hace progresivamente más complejo (y más eficiente) a medida que pasan los años. De esta forma el control inhibitorio, la memoria de trabajo y la flexibilidad mental, conformarían el primer sistema de FE (niñez); posteriormente se añadiría la memorización estratégica, y la planeación compleja (inicios de la adolescencia); en un tercer momento (a lo largo de la adolescencia), la actitud abstracta y el procesamiento psicolingüístico complejo.

En la medida en que los niños crecen, su capacidad para comprender relaciones complejas se incrementa, el incremento en la complejidad ocurre por el aumento en el número y grado de complejidad de los elementos incluidos en el sistema de relación que se construye (Kerr \& Zelazo, 2003). Se plantea que es posible evaluar el desarrollo en la complejidad de procesamiento de los niños, al determinar el número y las características de los elementos y reglas que incluyen en los modelos de razonamiento que construyen para resolver un problema (una mayor capacidad de memoria de trabajo, permite la posibilidad de representar-incluir mayores elementos durante un análisis complejo). La capacidad para construir sistemas de elementos-reglas más complejos permiten una selección más flexible de diferentes alternativas 
que compiten entre sí, y también posibilitan procesar los elementos de un problema dado tanto de forma paralela (analizando cada uno de los elementos) como jerárquica, lo que permite integrar elementos básicos en sistemas de razonamiento complejo (Zelazo et al., 2003).

El número y la complejidad de los elementos incluidos en los sistemas de razonamiento se incrementan con la edad, ya a los 7 años de edad, los niños pueden construir reglas de relaciones ternarias (Andrews, Halford, Murphy \& Knox 2009). La integración FE-cognición se relacionaría con las capacidades que son más evidentes a partir de los 11 años de edad: pensamiento lógico, construcción de hipótesis y solución de problemas; y un desarrollo significativo de la capacidad de integración de información que requiere manejo del tiempo (en años y décadas) (Fuster, 2002).

\section{Escolaridad y actividad escolar}

El efecto de la escolaridad sobre el desempeño cognitivo ha sido ampliamente demostrado (Reis, Petersson, CastroCaldas \& Ingvar, 2001), excepto para las FE. Aplicando una batería neuropsicológica de pruebas de funciones ejecutivas (Flores Lázaro et al., 2012), a un grupo de jóvenes-adultos (23.69 años de edad en promedio) con sólo 10.6 años de escolaridad en promedio, y al compararlos con un grupo de jóvenes universitarios, y otro grupo de adolescentes cursando el bachillerato; Flores Lázaro et al. (2011) encontraron que en su mayoría las FE son más sensibles al efecto de la actividad escolar (por ejemplo estar cursando el bachillerato) que al número de años (nivel universitario) que se estudió. Los jóvenes con nivel de bachillerato que trabajan en empleos técnicos y no continuaron sus estudios universitarios, generan principalmente categorías semánticas de tipo funcional -descripción de la función concreta de los objetos-. En la Figura 3 se presenta el desempeño de un grupo de adolescentes escolarizados vs un grupo de jóvenes con nivel escolar de bachillerato (empleados en fábricas), y un grupo de jóvenes universitarios. Se observa que incluso el grupo de adolescentes que se encuentran cursando el bachillerato presenta un mayor número de categorías abstractas generadas.

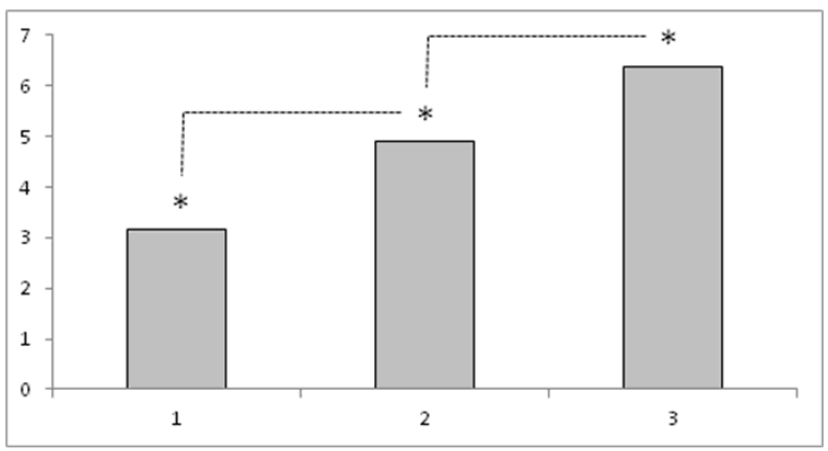

Figura 3. Generación de categorías abstractas.

1: Jóvenes con nivel de bachillerato, 2: Adolescentes cursando el bachillerato, 3: Jóvenes universitarios. Los asteriscos indican diferencias estadísticamente significativas (1 vs 2, 2 vs 3). (Adaptado de Flores Lázaro et. al. 2011).
De las $20 \mathrm{FE}$ que se estudiaron en total, sólo la Fluidez verbal (fluidez de verbos) fue específicamente sensible al nivel universitario. En la figura 4 se presenta el desempeño para la fluidez de verbos, se puede observar cómo los adolescentes que se encuentran cursando el bachillerato tienden a presentan un mayor desempeño (no estadísticamente significativo) que los jóvenes trabajadores; a su vez los universitarios presentaron el mayor desempeño de todos los grupos. Lo que indica que es el nivel universitario el que genera una mayor capacidad (número de verbos emitidos en un minuto).

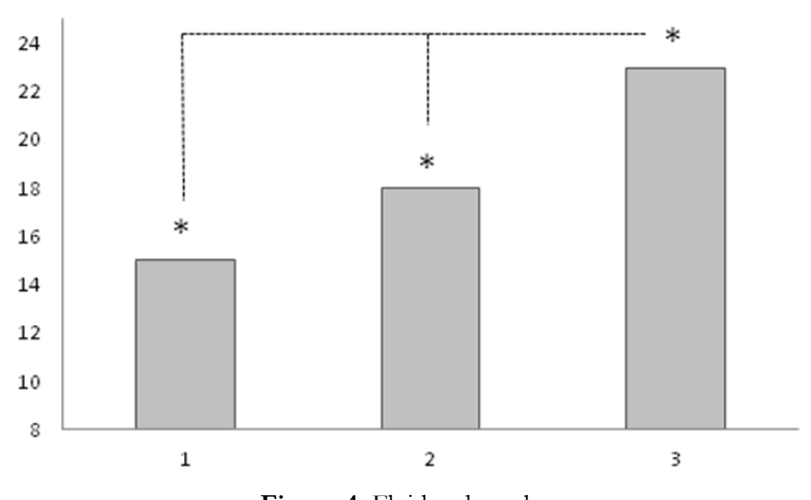

1: Jóvenes con nivel de bachillerato, 2: Adolescentes cursando el bachillerato, 3: Jóvenes universitarios. Se presentaron diferencias significativas entre el grupo 1 y el 3, y entre el grupo 2 y 3. (Adaptado de Flores Lázaro et. al. 2011).

\section{Factores adicionales}

Existen un número significativo de factores adicionales al efecto de la edad-escolaridad en el desarrollo de las FE, tales como los estilos parentales, el status socioeconómico, el bilingüismo, y los contextos culturales. Se ha encontrado que el nivel educativo de los padres tiene efectos significativos en el desempeño ejecutivo de los niños, se piensa que entre las principales causas se encuentra un hogar más enriquecido de estímulos académicos-intelectuales que promueven un mejor desarrollo cognitivo (Ardila, Roselli, Matute \& Guajardo, 2005). También se ha encontrado que los niños chinos se desempeñan mejor en pruebas de atención e inhibición que sus contrapartes norteamericanos (Lan, Legare, Ponitz, Li \& Morrison, 2011); así mismo los niños (al igual que los adultos) bilingües también presentan mejor desempeño en pruebas de atención y control inhibitorio, que los sujetos nobilingües (Bialystok \& Martin, 2004; Poulin-Dubois, Blaye, Coutya, \& Bialystok, 2011; Sarsour et al., 2011); se ha encontrado que en sujetos bilingües ambos idiomas se preactivan simultáneamente aun cuando sólo uno de ellos se está utilizando; se plantea que el tener que seleccionar entre los recursos lingüísticos de dos idiomas (conflicto de procesamiento) es el principal mecanismo de este mejor desempeño, ya que este efecto no se presenta para pruebas que requieren control de impulsos ante estímulos individuales (Carlson \& Meltzoff, 2008; Martin-Rhee; Byalistok, 2008). Ante pruebas 
de memoria de trabajo, los niños bilingües presentan mejor desempeño, siendo más evidente si la complejidad de la prueba requiere más aporte de otras FE (Morales, Calvo \& Bialystok, 2013). Estos resultados indican que el aprendizaje y desarrollo del bilingüismo provocan una mejor capacidad para la activación y coordinación de dos o más procesos cognitivos, mejorando el desempeño del control ejecutivo en la infancia.

También se han encontrado influencias en el autocontrol de los niños debido a estilos parentales de crianza (Bernier, Carlson \& Whipple, 2010; Sarsour et al., 2011), así como la influencia de los estilos parentales en el desarrollo de estrategias de aprendizaje autorregulado: mayor independencia para el aprendizaje (Pino-Pasternak \& Whitebrad, 2010).

\section{Conclusión}

Existe ya un número y una diversidad importante de estudios sobre el desarrollo de FE, que permiten construir un mapa conceptual inicial de su desarrollo a lo largo de la niñez y la adolescencia. En su gran mayoría comprueban el planteamiento de Anderson (2001), el cual podría ser la primera característica general de las FE: presentan un desarrollo secuencial, el cual es más intenso durante la infancia, reduciendo su velocidad a inicios de la adolescencia (conducta curvilínea). En su mayoría las FE terminan su desarrollo individual a inicios-mediados de la adolescencia. Las FE reciben un efecto diferencial de la escolaridad y sobre todo de la

\section{Referencias}

Anderson, V. (2001), Assessing executive functions in children: biological, psychological and developmental considerations. Pediatric Rehabilitation, 4, 119-136.

Anderson, P. (2002). Assessment and Development of Executive Function (EF) During Childhood. Child Neuropsychology, 8, 71-82.

Andrews, G. Halford, G. S., Murphy, K. \& Knox, K. (2009). Integration of weight and distance information in young children: The role of relational complexity. Cognitive development, 24, 49-60.

|Ardila, A. Roselli, M. Matute, E. \& Guajardo, S. (2005). The Influence of the Parents' Educational Level on the Development of Executive Functions. Developmental Neuropsychology, 28 (1), 539-560.

Armengol, C.G. (2002). Stroop test in Spanish children's norms. Clinical Neuropsychology, 16, 67-68.

Ávila, R. (2004). Lo que hacen los niños: verbos y grado escolar. Studia Romanica Posnaniensia, 31,129-146.

Bechara, A., Tranel, D., Damasio, H. \& Damasio, A. R. (1996), Failure to respond autonomically to anticipated future outcomes following damage to prefrontal cortex. Cerebral Cortex, 6, 215-25.

Bernier, A., Carlson, S. M., \& Whipple, N. (2010). From External Regulation to Self-Regulation: Early Parenting Precursors of Young Children's Executive Functioning . Child Development, 81, 326-339.

Best, J. R., Miller, P. H. \& Naglien, J. A. (2001). Relations between executive function and academic achievement from ages 5 to 17 in a large, representative national sample. Learning and Individual Differences, 21, 327-336.

Best, J. R., Miller, P. H. \& Jones, L. L. (2009). Executive Functions after Age 5: Changes and Correlates. Developmental Review, 29,180-200. actividad escolar, algunas de ellas son más sensibles que otras. Así mismo reciben efectos de la condición particular en el desarrollo del niño (cultura de crianza, bilingüismo, estilos parentales, etc).

El efecto que tiene el desarrollo de las FE sobre el desarrollo cognitivo es aditivo-sistémico a lo largo de la infanciaadolescencia: selectividad y control de los procesos cognitivos, mayor capacidad para crear esquemas mentales, mayor flexibilidad mental, incremento en el uso y complejidad de estrategias de memoria-aprendizaje, y mayor organización y planeación de la actividad cognitiva y conductual; en el fondo de un desarrollo constante de la actitud para analizar de forma cada vez más abstracta, así como de utilizar elementos psicolingüísticos cada vez más complejos.

Sin embargo aún falta información sobre la forma en cómo se integran entre ellas (sobre todo durante la adolescencia) y cómo se integran con otras capacidades cognitivas a lo largo del desarrollo. No existen datos mínimos sobre el desarrollo de FE durante la juventud, la literatura sólo sugiere que es un periodo "sin cambios significativos" (por ejemplo: Anderson, 2002; Best et. al., 2009; Luciana et al., 2005; Romine \& Reynolds, 2005), es muy probable que esto sea debido al bajo techo de complejidad cognitiva de las pruebas (neuropsicológicas) frecuentemente utilizadas.

Las FE son muy diversas, por lo que su estudio debería de basarse en un modelo de desarrollo, que incluya un número y diversidad significativa de ellas, pero sobre todo que sea conceptualmente representativo de su complejidad.

Best, J. R. \& Miller, P. H. (2010). A developmental perspective on executive function. Child Development, 81, 1641-60.

Bialystok, E. \& Martin, M. M. (2004). Attention and inhibition in bilingual children: evidence from the dimensional change card sort task. Developmental Science, 7(3), 325-339.

Bjorklund, D. F., Dukes, C., \& Brown, R. D. (2009). The development of memory strategies. In N. Cowan \& M. Courage (Eds.) The development of memory in infancy and childhood (2nd ed.) (pp. 145-175). New York, NY: Psychology Press.

Brocki, K. C. \& Bohlin, G. (2004). Executive functions in children aged 6 to 13: A dimensional and developmental study. Developmental Neuropsychology, 26, 571-593.

Boroditsky, L. (2007). Comparison and the development of knowledge. Cognition, 102, 118-28.

Bright, P., Moss, H., \& Tyler, L. K. (2004). Unitary vs multiple semantics: PET studies of word and picture processing. Brain and Language, 89, 417-32.

Cinan, S. (2006). Age-related changes in concept formation, rule switching, and perseverative behaviors: A study using WCST with 12 unidimensional target cards. Cognitive Development, 21, 377-382.

Crone, E. A. (2009). Executive functions in adolescence: inferences from brain and behavior. Developmental Science, 12, 825-30.

Crone, E. A., Bunge, S. A., Latenstein, H. \& van der Molen, M. W. (2005). Characterization of children's decision making: sensitivity to punishment frequency, not task complexity. Child Neuropsychology, 11, 245-63.

Crone, E. A., \& van der Molen, M. W. (2004). Developmental changes in real life decision making: performance on a gambling task previously shown to depend on the ventromedial prefrontal cortex. Developmental Neuropsychology, 25, 251-79. 
D’Amico, S., Devescovi, A., Bates, E. (2001). Picture naming and lexical access in Italian children and adults. Journal of Cognition and Development, 2 (1), $71-105$

Diamond, A. (2002). A model system for studying the role of dopamine in prefrontal cortex during early development in humans. En Johnson y Munakata, Gilmore (Eds.), Brain Development and Cognition (pp. 466-503.) Blackwell Publishers.

Diamond A. \& Lee K. (2011). Interventions shown to Aid Executive Function Development in Children 4-12 Years Old. Science, 19, 959-964.

De Marie, D., \& Ferron, J., (2003). Capacity, strategies, and metamemory: tests of a three-factor model of memory development. Journal of Experimental Child Psychology, 84, 167-93.

Delis D, Squire L. R., Bihrle, A. \& Massman, P. Componential Analysis of Problem-Solving Ability: Performance of Patients with Frontal Lobe Damage and Amnesic Patients on a New Sorting Test. Neuropsychologia 1992, 30, 683-697.

Elliot, R., Dolan, R. J., \& Frith, C. D. (2000). Dissociable functions in the medial and Lateral orbitofrontal cortex: Evidence from human neuroimaging studies. Cerebral Cortex, 10, 308-317.

Fernandez-Duque, D., Baird, J. A. \& Posner, M. (2000) Executive Attention and Metacognitive Regulation. Consciousness and Cognition, 9, 288-307.

Huizinga, M., Dolan, C. V., \& Van der Molen, M. W. (2006) Age-related change in executive function: Developmental trends and a latent variables analysis. Neuropsychologia, 44, 2017-203.

Hooper, C. J., Luciana, M., Conklin, H. M., \& Yarger, R. S. (2004) Adolescent performance on the Iowa Gambling Task: implications for the development of decision making and ventromedial prefrontal cortex. Developmental Psychology, 40, 1148-1158.

Kemmerer, D. \& Gonzalez Castillo, J. (2010). The two-level theory of verb meaning: an approach to integrating the semantics of action with the mirror neuron system. Brain and Language, 112 (1), 54-76.

Kerr, A. \& Zelazo, P. D. (2003). Development of "hot" executive functions, the children's gambling task. Brain and cognition, 55, 148-157.

Koriat, A., Goldsmith, M., Schneider, W. \& Nakash-Dura, M. (2001). The credibility of children's testimony:Can children control the accuracy of their memory reports? Journal of Experimental Child Psychology, 79, 405437.

Flores Lázaro, J. C., Ostrosky-Solís, F. (2008). Developmental characteristics in category generation reflects different prefrontal cortex maturation. Advances in Psychology Research, 55, 3-13.

Flores Lázaro, J. C., Tinajero Carrasco, B. \& Castro Ruiz, B. (2011). Influencia del nivel y de la actividad escolar en las funciones ejecutivas. Revista interamericana de Psicología, 45, 281-292.

Flores Lázaro J. C. \& Ostrosky-Shejet F., (2012). Desarrollo neuropsicológico de lóbulos frontales y funciones ejecutivas. México, D. F. Manual Moderno.

Flores Lázaro, J. C., Ostrosky-Shejet, F., \& Lozano Gutiérrez, A. (2012). BANFE: Batería Neuropsicológica de Funciones Ejecutivas y Lóbulos Frontales. México, D. F.: Manual Moderno.

Fuster, J. (2002), Frontal Lobe and Cognitive development. Journal of Neurocitology, 31, 373-285.

Garber, P., \& Golden-Meadow, S. (2002). Gesture Offers insight into problem-solving in adults and children. Cognitive Science, 26, 817-831.

Garon, N., Bryson, S. E., \& Smith, I. M. (2008). Executive function in preschoolers: A review using an integrative framework. Psychological Bulletin, $134,31-60$

Ghetti, S., Lyons, K., Lazzarin, F., \& Cornoldi, C. (2008). The development of metamemory monitoring during retrieval: The case of memory strength and memory absence. Journal of Experimental Child Psychology, 99, 157-181.

Gelaes, S., \& Thibaut, J. P. (2006). The role of the structure of parts and of the overall object shape in children's generalization of novel object names. Cognitive development, 21, 369-376.

Gentner, D., \& Namy, L. L. (2000). Comparison in the development of categories. Cognitive development, 14, 487-513.

Goldberg, E. (2001), The executive Brain, frontal lobes and the civilized mind. New York: Oxford University Press.

Huizinga, M., Dolan, C. V., \& Van der Molen, M. W. (2006) Age-related change in executive function: Developmental trends and a latent variables analysis. Neuropsychologia, 44, 2017-203.
Jia, G, Kohnert, K., Collado, J. \& Aquino-Garcia, F. (2006). Action Naming in Spanish and English by Sequential Bilingual Children and Adolescents. Journal of Speech, Language, and Hearing Research, 49 , 588-602.

Kerr, A., Zelazo, P. D. (2003). Development of "hot" executive functions, the children's gambling task. Brain and cognition, 55, 148-157.

Koriat, A., Goldsmith, M., Schneider, W., \& Nakash-Dura, M. (2001). The credibility of children's testimony: Can children control the accuracy of their memory reports? Journal of Experimental Child Psychology, 79, 405437.

Lan, X., Legare, C. H., Ponitz, C. C., Li, S. \& Morrison, F. J (2011). Investigating the links between the subcomponents of executive function and academic achievement: a cross-cultural analysis of Chinese and American preschoolers. Journal of Experimental Child Psychology 108, 677-92.

Lange, G., Guttentag, R. E., \& Nida, R. (1990). Relationships between study organization, retrieval organization, and general and strategy-specific memory knowledge in young children. Journal of Experimental Child Psychology, 49, 126-46.

Leon-Carrion, J., Garcia-Orza, J., Perez-Santamaria, F. J. (2004). Development of the inhibitory component of the executive functions in children and adolescents. International Journal of Neuroscience, 114, 1291-311.

Levin, H. S., Song, J., Swing-Cobbs, L. \& Roberson, G. (2001). Porteus maze performance following traumatic brain injury in children. Neuropsychology, 15, 557-67.

Lezak, M. D. (2004). Neuropsychological Assesment. (4ta ed). New York: Oxford University Press.

Luciana, M. \& Nelson, C. A. (2002). Assesment of neuropsychologycal function trough use of the cambridge neuropsychologycal testing automated battery: performance in 4 to 12 year old children. Developmental Neuropsychology, 22, 595-624.

Luciana M, Conklin H. M, Hooper C. J, \& Yarger R. S. (2005). The development of nonverbal WM and executive control processes in adolescents. Child Development, 76, 697-712.

Luria, A. R. (1986). Las Funciones Corticales Superiores del Hombre. México: Fontamara.

Martin-Rhee, M. \& Bialystok, E. (2008). The development of two types of inhibitory control in monolingual and bilingual children. Bilingualism: Language and Cognition, 11 (1), 81-93.

Masterson, J., Drukes, J. \& Galiene, D. (2008). Object and action picture naming in three- and five-year-old children. Journal of Child Language, 35 , 373-402.

Matute, E. Roselli, M. \& Ardila, A. (2004). Verbal and no verbal fluency in spanish speaking children. Developmental Neuropsychology, 26, 647-660.

Meltzoof, A. N. \& Carlson, S. M. (2008). Bilingual experience and executive functioniong in young children. Developmental Science, 11(2), 282-298.

Matute, E., Chamorro, Y., Inozemtseva, O., Barrios, O., Rosselli, M., \& Ardila, A. (2008). The effect of age in a planning and arranging task ('Mexican pyramid') among schoolchildren. Revista de Neurología, 47, 61-70

Morales, J., Calvo A., \& Bialystok, E. (2013). Working memory development in monolingual and bilingual children. Journal of Experimental Child Psychology, 114 (2), 187-202

Miller, P. H. (2000). How to best utilize a deficiency. Child Development, 71, 1013-1017.

Nelson, T. O. Narens, L. (1994) The role of metacognition in problem solving. In J. Metcalfe \& A. Shimamura (Eds.), Metacognition (pp. 207-226). Cambridge: MIT press.

Noppeney, U., Price, C. J., Penny, W. D. \& Friston, K. J. (2005). Two Distinct Neural Mechanisms for Category-selective Responses. Cerebral Cor tex. 16, 437-445.

Noppeney, U., \& Price C. J. (2002). A PET study of stimulus- and taskinduced semantic processing. Neuroimage. 15, 927-933.

Overman, W. H., Frassrand, K., Ansel, S., Trawlater, S., Bies, B. \& Redmond, A. (2004). Performance on the Iowa card task by adolescents and adults. Neuropsychologia, 42, 1838-1851. 
Pino-Pasternak, D. \& Whitebrad, D. (2010). The role of parenting in children's self-regulated learning. Educational Research Review, 5, 220-242.

Pineda, D.A., Merchan, V., Roselli M. \& Ardila A. (2000). Estructura factorial de la función ejecutiva en estudiantes universitarios jóvenes. Revista de Neurología, 31(12), 1112, 1118

Poulin-Dubois, D., Blaye, A., Coutya, J. \& Bialystok, E. (2011). The effects of bilingualism on toddlers' executive functioning. Journal of Expermental Child Psychology, 108, 567-579.

Prevor, M., \& Diamond, A. (2005). Color-object interference in young children: A Stroop effect in children 31/2-61/2 years old. Cognitive development, 20, 256-278.

Ramírez, M., Ostrosky-Solís, F., Fernandez, A. \& Ardila, A. (2005). Semantic verbal fluency in Spanish-speaking people: a comparative analysis Revista de Neurología, 41, 463-468.

Reis, A., Petersson, K. M. Castro-Caldas, A. \& Ingvar, M. (2001). Formal Schooling Influences Two but Not Three-Dimensional Naming Skills. Brain and Cognition, 47, 397-411.

Roebers, C. M. (2006). Developmental Progression in Children's Strategic Memory Regulation. Swiss Journal of Psychology, 65, 193-200.

Roebers, C. M., von der Linden, N., Schneider, W. \& Howie, P. (2007). Children's metamemorial judgments in an event recall task. Journal of Experimental Child Psychology, 97, 117-37.

Romer, D., Betancourt, L. M., Brodsky, N. L., Giannetta, J. M., Yang, W. \& Hurt, H. (2011). Does adolescent risk taking imply weak executive function? A prospective study of relations between working memory performance, impulsivity, and risk taking in early adolescence. Developmental Science, 14, 1119-33.

Rosso, I. M., Young, A. D., Femia, L. A. \& Yurgelun-Todd, D. A. (2004). Cognitive and emotional components of frontal lobe functioning in childhood and adolescence. Annals of the New York Academy of Science, 1021, 355-362.

Romine, C. B., \& Reynolds. C. R. (2005). A model of the development of frontal lobe functioning: findings from a meta-analysis. Applied Neuropsychology, 12, 190-201.

Sarsour, K., Sheridan, M., Jutte, D., Nuru-Jeter, A., Hinshaw, S. \& Boyce, W. T. (2011). Family socioeconomic status and child executive functions: the roles of language, home environment, and single parenthood. Journal of the International Neuropsychological Society, 17, 120-32.

Scerif, G. (2010). Attention trajectories, mechanisms and outcomes: at the interface between developing cognition and environment. Developmental Science, 13(6), 805-812

Schlagmuller, M. \& Schneider, W. (2002). The development of organizational strategies in children: evidence from a microgenetic longitudinal study. Journal of Experimental Child Psychology. 81, 298-319.

Schneider, W. (2002). Memory development in childhood. In U. Goswami (Ed.), Blackwell Handbook of Childhood Cognitive Development (pp. 236-256). U.K: Blackwell Publishers.

Schneider, W., Kron, V., Hünnerkopf, M., \& Krajewski, K. (2004). The development of young children's memory strategies: First findings from the Würzburg Longitudinal Memory Study. Journal of Experimental Child Psychology, 88, 193-209.
Shimamura A. P. (2000). Toward a cognitive neuroscience of metacognition. Consciousness and Cognition, 9, 313-323.

Siegler, R. S. (2004). Turning memory development inside out. Developmental Review, 24, 469-475.

Smidts, D. P., Jacobs, R. \& Anderson, V. (2004). The Object Classification Task for Children (OCTC): a measure of concept generation and mental flexibility in early childhood. Developmental Neuropsychology, 26, 385401.

Spalek K, Thompson-Schill S. L. (2008). Task-dependent semantic interference in language production: an fMRI study. Brain and Language, 107(3), 220-8.

Thibaut, J. P., French, R. \& Vezneva, M. (2010). Cognitive load and executive functions, Journal of Experimental Child Psychology, 106, 1-19.

Thordardottir, E. T. \& Weismer, S. E. (2001). High-frequency verbs and verb diversity in the spontaneous speech of school-age children with specific language impairment. International Journal of Language and Communicative Disorders, 36, 221-224.

van den Heuvel O. A., Groenewegen, H. J., Barkhof, F., Lazeron, R., van Dyck, R. \& Veltman D. J. (2003). Frontostriatal system in planning complexity: a parametric functional magnetic resonance version of tower of london task. Neuroimage, 18, 367-374.

Van Leijenhorst, L., Westenberg, P. M. \& Crone, E. A. (2008). A developmental study of risky decisions on the cake gambling task: age and gender analyses of probability estimation and reward evaluation. Developmental Neuropsychology, 33, 179-96.

Vigliocco, G., Vinson, D. P. Damian, M. F. Levelt, W. (2002). Semantic distance effects on object and action naming. Cognition, 85, B61-B69.

Waters, H. S. (2000). Memory strategy development: do we need yet another deficiency? Child development, 71, 1004-1012.

Welsh, M. C. (1991). Rule-guided behavior and self-monitoring on the Tower of Hanoi disk-transfer task. Cognitive Development, 6, 59-76.

Wright, B. C. \& Wanley, A. (2003). Adults' versus children's performance on the Stroop task: interference and facilitation. British Journal of Psychology, 94, 475-485.

Zelazo, P. D. \& Frye, D. (1997). Cognitive complexity and control: A theory of the development of deliberate reasoning and intentional action. En N. Stamenov (Ed.), Language structure discourse, and the access to consciousness (pp. 113-153). Amsterdam: John Benjamins.

Zelazo, P. D., \& Cunningham, W. (2007). Executive function: Mechanisms underlying emotion regulation. In J. Gross (Ed.), Handbook of emotion regulation (pp, 135-158). New York: Guilford Press.

Zelazo, P. D., Muller, U. (2002). Executive function in typical and atypical development. En U. Goswami (Ed.), Handbook of child Cognitive development (pp. 445-469). Oxford: Blackwell.

Zelazo, P. D., Muller, U., Frye, D. \& Marcovitch, S. (2003). The development of executive function in early childhood. Monographs of the Society for Research in Child Development, 68, 91-119.

(Articulo recibido: 10-7-2012; revisado: 15-5-2013; aceptado: 1-6-2013) 